\title{
Bidi smokers at increased risk of oral cancer
}

\author{
Do bidi smokers have an increased risk for oral cancer?
}

\begin{abstract}
Rahman M, Sakamoto J, Fukui T. Bidi smoking and oral cancer: a meta-analysis. Int J Cancer 2003 10; 106:600-604
\end{abstract}

Data sources Source articles were searched for using Medline, the Cochrane Library and within the references lists of identified articles.

Study selection Articles were selected that included data enabling construction of $2 \times 2$ tables to estimate odds ratios (OR) and $95 \%$ confidence intervals $(\mathrm{Cl})$.

Data extraction and synthesis For each study, two-way contingency tables were constructed, based on exposure frequency distributions, for cases and controls. Unadjusted OR and $95 \% \mathrm{Cl}$ were recalculated based on the reported data using standard procedures. Separate contingency tables were made for bidi smoking, cigarette smoking and both types of smoking if the data were available in the same article. The overall OR combined across all studies, and its $95 \% \mathrm{Cl}$, was calculated using a random-effects model for bidi and cigarette smoking. Tests for publication bias and heterogeneity were conducted. Confounding factors, for example, betel quid chewing or alcohol use, were not included in the meta-regression model.

Results An increased risk of oral cancer was found for bidi smokers compared with people who had never smoked (OR, 3.1; 95\% Cl, 2.05.0) whereas no significant pattern of risk was found for cigarette smokers (OR, $1.1 ; 95 \% \mathrm{Cl}, 0.7-1.8)$. There was substantial heterogeneity in the pooled OR estimate.

Conclusions The results clearly indicate that bidi smokers are at increased risk of oral cancer. It is important that this information be incorporated into smoking prevention and cessation efforts, particularly in the urban poor and rural mass in south Asian countries where bidi smoking is widespread.

\section{Commentary}

The data for this review come from case-control studies examining the role of bidi smoking in oral cancer that were conducted in India along with one multicentre study from south Asia. Twelve such studies, carried out from 1966 to 2002, were included, but the metaregression model did not take account of confounding factors such as use of areca nut and smokeless tobacco in betel quid. That an increased risk of oral cancer was found for bidi smokers compared with people who had never smoked but not for cigarette smokers is surprising because cigarette smoking is an established risk factor for oral cancer. There was great heterogeneity in the pooled OR estimate particularly because of variability in the number of cases in the included studies.

This review shows that bidi smokers have an increased risk of oral cancer. The authors highlight the importance of incorporating this information into smoking prevention and cessation efforts, especially for men in urban poor and rural areas in south Asian countries where bidi smoking is common. Bidi smoking has also been reported to be associated with a significantly higher mortality compared with tobacco chewing. ${ }^{1}$ As Asian immigrants may continue to smoke bidis - a product now available in Europe and Americas - in their new settlements, these findings are also of relevance to primary care providers in industrialised countries. It is interesting to note that brief interventions in dental settings have been shown to result in quiting tobacco use of $5-12 \%$ of users. ${ }^{2,3}$

\section{Practice point}

- Bidi smoking increases the risk of oral cancer. This is not only relevant for preventive efforts in south Asian countries but in Europe and the Americas where bidis are increasingly available.

\section{Saman Warnakulasuriya \\ Guy's, Kings and St Thomas' Dental Institute, Kings College London, London, UK}

1. Gupta PC, Mehta FS, Irani RR. Comparison of mortality rates among bidi smokers and tobacco chewers. Ind J Cancer 1980; 17:149-152.

2. Warnakulasuriya $S$. Effectiveness of tobacco counselling in the dental office: an overview. I Dent Ed 2002; 66:1080-1089.

3. Brothwell DJ. Should the use of smoking cessation products be promoted by dental offices? An evidence-based report. J Can Dent Assoc 2001; Mar:67:149-67:155.

Evidence-Based Dentistry (2005) 6, 19.

doi:10.1038/sj.ebd.6400297 